\title{
Towards Self Sustainable Railway Transportation System!
}

\author{
Axay Upadhyay \\ PG Student, \\ PIET, Baroda \\ Gujarat, India
}

\author{
Rahul Joshi \\ Asst. Professor \\ PIET, Baroda \\ Gujarat, India
}

\begin{abstract}
This paper is focused on numerous challenges holding by Railway Transportation systems and various techniques to solve problems. Scheduling is one of the challenges in Railway transportation system which depends on numerous constraints. This paper consists of discussion of deadlock free schedule using Agent based system and expert system for the railway transportation system to minimize overall system delay by considering other constrain depend on this. Despite having Persist enhancement in approaches still have limitations which require improvement to meet the objective of the entire railway transportation system.
\end{abstract}

\section{General Terms}

Scheduling, Railway Transportation system Issues, Problem Models.

\section{Keywords}

Scheduling, rescheduling, Railway Transportation system, Agents Based system

\section{INTRODUCTION}

Merchandise and passengers using the transportation system since long ago railway is one of them. The railway system management having four categories, which are strategic, tactical, operational and scheduling. There are two different aspects of any railway system which are Allocation \& scheduling and Monitoring \& Control. [5] Motivation behind this is increased demand in Railway transportation system holding numerous challenges in tactic railway operations may be scheduling, rescheduling, routing, energy optimization and other which are mentioned in the related work section. Multi agent based system consists of two layers regulating layer and learning layer one is to reschedule disrupted railway traffic with overall system delay constrain. While learning layer enhance the autonomy and intelligence of the whole system, when new disruption resolved by regulating layer learner agent receive message from every agent of regulating layer append prospective rule based if similar rule not found. Which can apply in scheduling, system control, conflict detection, signaling, load-balance and passenger convenience issue of railway transportation system. While Expert system solves problems such as expert tasks like Fault finding, design, manufacturing planning and more of engineering field that are normally solved by human experts. [73] Which concern with the Decision support system, intelligent transport system to deal with planning, control, maintenance, monitoring of the whole system. In the next section of existing method are mentioned Integer programming, mixed integer programming, petri nets, alternative graph, simulation model and other models as well as Expert system, agent based system used to solve numerous issue of railway transportation system. Limitation of existing methods to meet the objective of the railway transportation system are mentioned. Issues that need to solve for the resolution of human expert resolution are concluded at last.

\section{RELATED WORK}

This section consists of discuss related to some published research in this area of railway transportation system and literature that address issues of the railway transportation system.

Routing which considers as a strategic operational issue of railway transportation system addressed by an ant based Heuristic for the Railway travelling salesman problem to minimize overall travelling time of journey. Also for timetabling optimization of single and double track railway network. After that an efficient hybrid algorithm approach for to solve periodic single track train timetabling. After that MILP based approach for optimal train routing and scheduling for managing traffic perturbation in complex networks. [45] [46] [58] [71]

For energy efficiency of the railway transportation system In $2014 \mathrm{Su}$, Tang, Li, Gao proposed integrated algorithm for globally optimal operational schedule with better energy savings. [27] In 2013 Cardador, Cucala, Scire proposed energy regulation algorithm to get back train on schedule after delay in high speed train. [28] In $2013 \mathrm{Wu}$, Yan, Chen Proposed two algorithms for reduction of power consumption in wireless sensor network for railway disaster prevention and safety monitoring. [67]

For a tactic category operation like scheduling of railway transportation system in 1998 Jnaczura published thesis on Modelling and analysis of Railway network control logic using colored Petri nets. [76] In 2008 Tormos with another five proposed genetic algorithm for the train scheduling problem. [31] In 2011 Cleark proposed genetic algorithm with six others for Railway platform allocation. [57] In 2012 Bayhan with one other proposed simulation model approach for a feasible timetable generator framework for railway scheduling problem.[30] In 2013 Corman with other three proposed iterative optimization framework for railway schedule using alternative graph method. [29] In 2014 Ariano with three other proposed MILP based AGLIBRARY advanced technique for practical real time scheduling and evaluate applicability in railway. [35]

While In 2007 Tormos with four other proposed intelligent train scheduling on highly loaded network using a sequential algorithm and CSP approach.[32] In 2013 Zheng, Ling, Shi, Chen, Chen proposed hybrid biogeography based 
optimization algorithm for railway wagon scheduling in emergency relief. [69]

For deadlock in railway scheduling. In 2003 Fanti proposed deadlock prevention method for railway network using monitors for colored petri nets. [60] Zarnay proposed model of deadlock state of railway station operation using colored Petri nets. [15] In 2011 Vincent van der Vlies published thesis on Rail Transport Risks and Urban Planning: Solving deadlock situations between urban planning and rail transport of hazardous materials in the Netherlands. [75] In 2014 Ferrari with two other proposed model checking approach for deadlock free train schedule for management of critical section. [11] In 2014 Ferrari with three other proposed model checking approach for deadlock avoidance in train scheduling. [16] In $2014 \mathrm{Li}$, sheu, Gao proposed conflict distribution prediction method heuristic method for deadlock analysis, prevention and train optimal travel mechanism in single track railway system. [17]

For scheduling with passenger convenience In 2009 Xie, Luo, Peng proposed approach for optimization of train operating schedules of high speed intercity train for passenger convenience. [49] In 2014 Mesa and other proposed rescheduling railway timetable in presence of passenger transfer in between lines within transportation network. [40] Rescheduling require in case of flood and other emergency [63]

For uncertainty and delay In 2014 Corman with other four proposed optimal train schedule using branch and bound, FIFO for disturbance and uncertainty management. In 2011 Canca and three other proposed MINLP approach for different objectives in the determination of train scheduling. [33] For the sensitivity of scheduling, in 2008 castillo with three other proposed bisection method based on timetabling optimization of a single track railway with sensitivity analysis. [42] In 2014 Abid and Khan proposed Branch and bound method for sensitivity analysis of the train schedule of the railway track network. [13]

In 2006 ingolotti with another five proposed new heuristic to solve constrained specification for planning scheduling and decision support. [43] In 2015 Jiang and other two proposed train timetable forming quality evaluation based on the data envelopment analysis approach. [47] In 2007 Liebchen and mohring proposed the modelling power of periodic event schedule problem for railway timetable and planning. [48]

For the conflict in schedule, in 2011 Wen proposed approach for train operations conflict management of the high speed Railway. [59] In 2012 corman with other 3 proposed alternative graph based approach for Bi-objective conflict detection and resolution in railway traffic management. [14] In 2013 Larsen proposed approach for sustainability of optimal train schedule for disturbances. [18]

Railway scheduling requires to address delay for this, in 2010 muralli with three proposed delay estimation technique for single and double track railway. [20] In 2010 Goverde proposed bucket based delay estimation algorithm for delay propagation in large scale railway traffic network. [21]

For dispatch, in 2010 corman published thesis for real time railway traffic management for dispatching in complex, large and busy railway network. [74] In 2012 Kraseman proposed effective algorithm for fast response to reschedule railway traffic during the disturbance. In 2014 corman and other three proposed heuristic algorithm for dispatching and coordination in multi area railway traffic management. [23] In 2013 corman with two other proposed Roma Egtran approach and framework for stability analysis of railway dispatching plans in a dynamic environment. [24] In $2015 \mathrm{Xu}$, li yang proposed approach based on discrete event model-DEM for scheduling heterogeneous traffic on double track with efficient dispatching rules. [25]

After discussing work related to scheduling next is related to work proposed, survey and reported related rescheduling. For rescheduling, in 2005 Nirio and two other proposed train rescheduling algorithm based on PERT and Meta heuristic approach for passenger convenience. [37]

In 2011 Narayanswami and Rangaraj proposed review of expository analysis of railway scheduling and rescheduling approach. [44] In 2012 Yang proposed approach for rescheduling train with scenario based fuzzy recovery time representation. [66] In 2014 Cacchiani with another six published overview of recovery models and algorithm for real time railway rescheduling. [36] While In 2013 Albrecht and lee proposed problem space search meta heuristic approach for rescheduling rail network with maintenance and disruption. [41]

In 2012 Fan and other two have compare brute force, FCFS, tabu search, Genetic algorithm, simulated annealing, ant colony optimization, decision tree elimination for minimizing delay cost in disturbed the railway traffic scenario. [22] In 2013 Aranda and Rodenas proposed demand based weighted train delay approach for rescheduling railway network in real time using an alternative graph method. [19]

In 2013 Dundar proposed train rescheduling using genetic algorithm and artificial neural network. [38] In 2014 Almodovar and Rodenas proposed two greedy heuristic approaches for online reschedule optimization for passenger in case of emergency. [39] In 2015 wei fang with two other published survey paper on rescheduling problem models and solution by models. [1]

While In 2013 bergmier with three other study use of machine learning methods for incident prediction in high speed train tracks. Decision support system, expert system and intelligent transport introduce to address numerous issues of railway transportation system. [65] For this In 2010 Zak published a chapter on decision support system in transportation. [61] In 2011 Corman with three other propose branch and bound based decision support system for optimal multi class rescheduling in the railway network. [34] In 1995 Larroche proposed a real time expert system that automates rail routes. [50] In 2006 tsamboulas and Mikroudis proposed Trans-pol which is decision support system and an expert system for the transportation system. [68] Ingoloti and four others have proposed a decision support system for railway scheduling problems. [26] In 2011 jiang and other two proposed sustainable strategies for railway intelligent transport system using Artificial intelligence. [55] In $2013 \mathrm{He}$, song and Chaudhary proposed service oriented intelligent group decision support system application in the transportation system. Li, Jia, Nie study railway intelligent transport system architecture. [56]

Up to now work related to routing, energy optimization, scheduling, rescheduling of the railway system by numerous methods as well as decision support system, expert system 
and intelligent transportation system to address numerous issues of the railway transportation system. After that, work related to the railway transportation system issue using multi agent system. For this Mascardi with four other proposed the multi agent system for monitoring and diagnosis of railway signaling. [9] In 2004 proenca and oliveira proposed multi agent railway control system which consist of two layers: Regulating Layer and Learning Layer"Control" layer is responsible for traffic supervision, regulation, security and fluidity, including three distinct agent types: "Supervisor", "Train" and "Station". The "Learning" layer, using situations accumulated by the "Control" layer, will infer rules that can improve traffic control processes, minimizing waiting time and stop orders sent for each train. In this future conflicts are harder to solve. [6] In 2005 basra and three other proposed multi agent system to resolve scheduling issue of the London underground. [7]

In $2007 \mathrm{Zi}-\mathrm{yu}$, lei proposed IDSS of high speed railway based on multi agent. [54] In 2008 Letia with other two proposed multi agent approach for distributed scheduling for real time railway traffic control. [51] Wang proposed agent based monitoring architecture for load balance in China train control system. [52] In 2010 sun with two other proposed multi agent based intelligent transport system for distributed hierarchical control for railway passenger dedicated line. [53]

In 2013 Ghosh with three other proposed multi agent system based simulation to improve Indian railway efficiency which consists of a Max-sum algorithm to minimize overall system delay and discuss six different cases where chance of conflict is possible at last they have discuss result comparison with max sum algorithm and without for delay parameter. [4] In 2014 Ankit Bhardwaj and two other proposed multi agent based train passing in railway system with minimum delay. [3]

In 2014 Anshul verma and pattnaik proposed mobile agent based train control system for mitigating meet conflict at the turnout. [8] In 2014 kuznetsov and three other proposed designs and optimization of algorithm for multi agent railway control system. [10] In 2015 Narayanswami and Rangaraj proposed multi agent system architecture for dynamic real time rescheduling and learning applied to railway transport. This paper has a multi agent based architecture for dynamic and real time rescheduling in case of conflict. A Multi agent system with 2 layers: Regulating layer which consists of four agents named train agent, supervisor agent, station agent, auctioneer agent and learning layer which having learner and facilitator as two different agents. Regulating layer control the conflict and learning layer generate or select a rule from rule based. [2]

\section{DISCUSSION}

There are several methods used to solve numerous issues and challenges of the railway transportation system which is categories into four different levels as per table I.

Table 1. Railway Operations Classification

\begin{tabular}{|l|l|}
\hline Category & Operation \\
\hline Strategic & Routing, energy efficiency \\
\hline Tactic & $\begin{array}{l}\text { Scheduling, traffic } \\
\text { management }\end{array}$ \\
\hline
\end{tabular}

\begin{tabular}{|l|l|}
\hline Real time control & Shunting, signaling \\
\hline Operational & Maintenance planning \\
\hline
\end{tabular}

In table I name of the operation of various categories which are discussed in this paper there are many other operation names which fall under various category are not listed there in table.

\subsection{Alternative Graph Model}

Alternative graph model conflicts free schedule with minimum delay in real time network. Conflict occurs when two or more trains require same block at the same time. Alternative graph model consist of a set of nodes, set of fixed arc and set of pairs of alternative arcs with positive, null or negative length. In Alternative Graph model for one route, one arc selected from each pair of alternative arc and set of arc from alternative arc.

\subsection{Discrete Event Model}

For conflict resolution within time bound and recover schedule from delay in the railway transportation system the discrete event model with genetic algorithms (GAs), simulated annealing (SA), and tabu search (TS).

\subsection{Mixed Integer Programming Model}

Mixed integer programming model is the base for MILP and MINLP model from the operational research point of view. Which consist of arrival, departure time and delay as decision variables in railway scheduling problem. The Mixed Integer Non Linear Programming model not more in use as it considers the objective function and constraints of the model are nonlinear.

\subsection{Petri Nets}

Petri nets to model for Train dispatch decision that require experience and a set of IF-THEN based knowledge rules. Colored Petri nets and fuzzy Petri nets were modelled by Petri nets.

\subsection{Simulation Model}

To support real time dispatching in rescheduling to conflict resolution and also to forecast the future status of the railway transportation system simulation model used to represent components of the railway system network.

\subsection{Expert System}

Expert system used to solve complex problem of a system that can be solved by human experts with experience. This requires expert level knowledge of the system to address the challenges of the system. Expert system deal with highly diverse problems. User, knowledge and interface are three components of expert systems. 


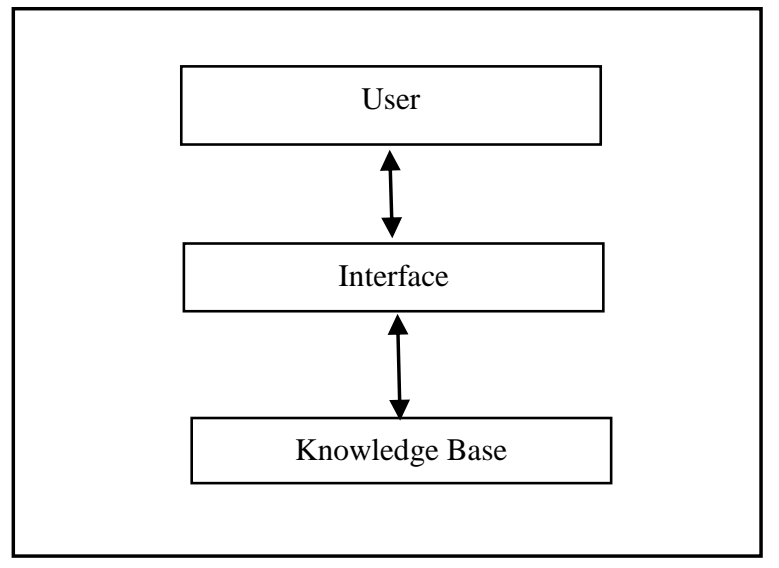

Figure 1. Component of Expert System [72]

\subsection{Agent Based System}

Agent based system consists of two layers in architecture which is regulating or control layer and the other is learning layer. Regulating or control layer consists of four agents in the layer, while learning layer consist of two agents leaner and facilitator. Supervisor remains active all time and sense disruption while auctioneer identifies conflicting trains for disruption and remain active until disruption solve. Train agent computes bids station agent verifies bids. When disruption solves rule updated to rule based and facilitator provides matching rule from the rule based on learning layer using Apriori algorithm of data mining. [2]

Table 2. Comparison of Problem Model

\begin{tabular}{|l|l|l|}
\hline \multicolumn{1}{|c|}{ Model } & \multicolumn{1}{|c|}{ Work } & Objective \\
\hline $\begin{array}{l}\text { Discrete Event } \\
\text { Model }\end{array}$ & $\begin{array}{l}\text { Dynamic model, } \\
\text { State of each } \\
\text { instance at a time }\end{array}$ & $\begin{array}{l}\text { Conflict Detection } \\
\text { and Recover } \\
\text { schedule from } \\
\text { delay }\end{array}$ \\
\hline Alternative Graph & $\begin{array}{l}\text { Graph based } \\
\text { Model with set of } \\
\text { Arc, edge, vertices }\end{array}$ & $\begin{array}{l}\text { Conflict schedule } \\
\text { with minimum } \\
\text { delay }\end{array}$ \\
\hline $\begin{array}{l}\text { Mixed Integer } \\
\text { Programming } \\
\text { Model }\end{array}$ & $\begin{array}{l}\text { Binary, Non } \\
\text { binary decision } \\
\text { Variable }\end{array}$ & $\begin{array}{l}\text { Dispatch } \\
\text { Decision, System } \\
\text { control }\end{array}$ \\
\hline Simulation Model & $\begin{array}{l}\text { Computer based } \\
\text { simulation from } \\
\text { set of components }\end{array}$ & $\begin{array}{l}\text { Rescheduling } \\
\text { conflict resolution, } \\
\text { dispatching. }\end{array}$ \\
\hline
\end{tabular}

\section{PROBLEM DESCRIPTION}

The railway transportation system across the globe having very large passenger flow and other freight. Among that global railway Indian Railways (IR) is a great national asset. A single transport network connects far flung areas of the country. It is one of the largest transportation and logistics network of the World which runs 19,000 trains. It runs 12,000 trains to carry over 23 million passengers per day connecting about 8,000 stations spread across the sub-continent. It is equivalent to moving the entire population of Australia. It runs more than 7,000 freight trains per day carrying about 3 million tonnes of freight every day. Its network of 65,000 route kilometres is more than one and half times the circumference of the earth. The biggest challenge facing Indian Railways today is its inability to meet the demands of its customers, both freight and passenger.

Apart from the quantum of investment, quality of delivery is also an issue. Cleanliness, punctuality of services, safety, quality of terminals, capacity of trains, quality of food, security of passengers and ease of booking tickets are issues that need urgent attention. Indian Railways have a glorious past, a turbulent present and a bright future. It is a giant emerging out of a deep slumber. An awake, alive and kicking Indian Railways can lead the country to greater heights of accomplishment. However, today, it is mired in a state of ennui, a state of cynicism that things cannot change. Its network is congested and finances are not easy to come by. Resources for development and replacement are stressed. It is finding it difficult to even meet its operational expenses. But, the spirit is still alive. To make an attempt at resurrecting Itself, Indian Railways have drawn up an ambitious five year action plan. The realization is there that if the vicious cycle of underinvestment is to be turned into a virtuous cycle of prosperity; crutches of support will have to be abandoned.

The Indian Railways will have to generate its own resources for its development. The next five years should change the face of Indian Railways. Faster trains, modern trains, swanky stations, skilled staff, should be the Railways of tomorrow. IR looks forward to becoming the nation's carrier and a multimodal integrator; making travel affordable, happy, convenient and reliable - a world class experience! IR also looks forward to becoming self-sustainable! [77]

\section{CONCLUSION}

From this discussion of railway transportation system conclusion made that from many years research work were proposed, survey and reviews were done and other report and thesis were published to address numerous challenges. Energy optimization, routing, scheduling- rescheduling with disruption handling, conflict detection and resolution, delay minimization, passenger convenience, uncertainty management, coordination management were addressed by existing methods which are a mixed integer programming model, alternative graph method, discrete event model, simulation model, Petri nets model, expert system and agent based system. As mentioned in previous section challenges that need to address are of resource allocation and management, applicability in large and congested network, passenger convenience.

Which require to resolve human expert resolution to meet the objective of the railway transportation system of India as well as applicable to the global railway transportation system also. Integration of approached used in existing methods can address some of the challenges of Indian railway which can be applied to other country also. If consider issues in the scheduling the required system that will address challenges like deadlock free schedule with a minimum system delay, expert dispatching and coordination to resolve conflict detection and accidents as well as applied in real time highly 
congested network for single as well as double track as per types of trains.

For emergency or in disruption situation this schedule requires reschedule that restores schedule of the system. Coordination with last train fault diagnosis require in this. [62], [64] Expert system addressing complex problems of a system which help in decision support system as well as in intelligent system. While multi agent system address problem of dynamic disruption for scheduling- rescheduling as well as control system, delay minimization, conflict, signaling, load balance and passenger convenience.

\section{REFERENCES}

[1] Wei Fang, Member, IEEE, Shengxiang Yang, Senior Member, IEEE, and Xin Yao, Fellow, IEE “A Survey on Problem Models and Solution Approaches to Rescheduling in Railway Networks" IEEE transaction on intelligent transaction systems 2015, IEEE.

[2] Sundravalli Narayanswami, Narayan Rangaraj “A MAS architecture for dynamic, real time rescheduling and learning applied to railway transportation" Expert system with applications 42, 2015, science direct, Elsevier 2015.

[3] Ankit Bhardwaj, Vikas Kumar Singh, Pankaj Kumar "Multi-Agent based Train Passing in Railway System with Minimum System Delay” IEEE 2014.

[4] Supriyo Ghosh, Animesh Dutta, Viviana Mascardi, and Daniela Briola, "Exploiting MAS-Based Simulation to Improve the Indian Railways' Efficiency" MATES 2013, LNAI 8076, Springer Verlag berlin, Heidelberg 2013.

[5] Martin p̌eni cka "Formal approach to Railway application” LNCS 4700, pp. 504-520, 2007, SpringerVerlag Berlin Heidelberg 2007.

[6] Hugo Proenca and Eug'enio Oliveira "MARCS - Multi Agent Railway Control System", IBERAMIA 2004, LNAI 3315, pp. 12-21, 2004. Springer-Verlag Berlin Heidelberg 2004.

[7] Rajveer Basra, Kevin Lü, George Rzevski, and Petr Skobelev "Resolving Scheduling Issues of the London Underground Using a Multi-agent System”, LNAI 3593, pp.188-196, Springer-Verlag Berlin Heidelberg 2005.

[8] Anshul Verma, K. K. Pattanaik "Mobile agent based train control system for mitigating meet conflict at turnout" Elsevier procedia computer science (32) 317324, 2014.

[9] Viviana Mascardi, Daniela Briola, Maurizio Martelli, Riccardo Caccia, Carlo Milani "Monitoring and Diagnosing Railway Signalling with Logic-Based Distributed Agents".

[10] N.A.Kuznetsov, I.K.Minashina, F.F.Pashchenko , N.G.Ryabykh, E.M.Zakharova "design and analysis of optimization algorithms for multi-agent railway control system", The 5th International Conference on Emerging Ubiquitous Systems and Pervasive Networks, Procedia Computer Science 37 ( 2014 ) 211 - 219 , Elsevier.

[11] Franco Mazzanti, Giorgio Oronzo Spagnolo, and Alessio Ferrari, "Designing a Deadlock-Free Train Scheduler: A Model Checking Approach ”NFM 2014, LNCS 8430, pp.
264-269, 2014. Springer International Publishing Switzerland 2014.

[12] Johanna Törnquist Kraseman "Design of an effective algorithm for fast response to the re-scheduling of railway traffic during disturbances" Transportation Research" Part C 20 (2012) 62-78 Elsevier.

[13] Malik Muneeb, Abid \& Muhammad Babar Khan "Sensitivity analysis of train schedule of a railway track network using an optimization modeling technique", 9 November 2014, springer.

[14] Francesco Corman, Andrea D'Ariano, Dario Pacciarelli, Marco Pranzo "Bi-objective conflict detection and resolution in railway traffic management Transportation" Transportation Research Part C 20 (2012) 79-94, Elsevier.

[15] Michal Zarnay "solving deadlock states in model of railway station operation using coloured petri nets".

[16] Franco Mazzanti, Giorgio Oronzo Spagnolo,Simone Della Longa, and Alessio Ferrari "Deadlock Avoidance in Train Scheduling: A Model Checking Approach" FMICS 2014, LNCS 8718, pp. 109-123, 2014, Springer International Publishing Switzerland 2014.

[17] Feng Li , Jiuh-Biing Sheu , Zi-You Gao "Deadlock analysis, prevention and train optimal travel mechanism in single-track railway system" Transportation Research Part B 68 (2014) 385-414 Elsevier.

[18] Rune Larsen, Marco Pranzo, Andrea D'Ariano, Francesco Corman, Dario Pacciarelli "Susceptibility of optimal train schedules to stochastic disturbances of process times" Springer Science + Business Media New York 2013.

[19] Jose Luis Espinosa-Aranda, Ricardo García- Ródenas “A demand-based weighted train delay approach for rescheduling railway networks in real time Journal of Rail Transport" Planning \& Management 3 (2013) 1-13 Elsevier.

[20] A delay estimation technique for single and double-track railroads Pavankumar Murali, Maged Dessouky, Fernando Ordóñez, Kurt Palmer "A delay estimation technique for single and double-track railroads" Transportation Research Part E 46 (2010) 483-495 Elsevier.

[21] Rob M.P. Goverde "A delay propagation algorithm for large-scale railway traffic networks Transportation" Research Part C 18 (2010) 269-287 Elsevier.

[22] Bo Fan, Clive Roberts, Paul Weston “A comparison of algorithms for minimising delay costs in disturbed railway traffic Scenarios", Journal of Rail Transport Planning \& Management 2 (2012) 23-33 Elsevier.

[23] F.Corman, A.D'Ariano, D.Paccia.relli c,n, M.Pranzo "Dispatching and coordination in multi-area railway traffic management" Computers \&Operations Research44 (2014)146-160 Elsevier.

[24] Egidio Quaglietta, Francesco Corman b,c, Rob M.P. Goverde "Stability analysis of railway dispatching plans in a stochastic and dynamic environment", Journal of Rail Transport Planning \& Management (2013), Elsevier 
[25] Xiaoming Xu, Keping Li, Lixing Yang "Scheduling heterogeneous train traffic on double tracks with efficient dispatching rules", Transportation Research Part B 78 (2015) 364-384, Elsevier .

[26] L. Ingolotti, P. Tormos ,A. Lova, F. Barber, M.A. Salido and M. Abril, "a decision support system (DSS) for the railway scheduling problem"

[27] Shuai Su, Tao Tang, Xiang Li, and Ziyou Gao, "Optimization of Multi train Operations in a Subway System", ieee transactions on intelligent transportation systems, vol. 15, no. 2, april 2014.

[28] C. Sicre, A.P.Cucala , A.Fernández-Cardador, "Real time regulation of efficient driving of high speed trains based on a Genetic algorithm and a fuzzy model of manual driving", Engineering Applications of Artificial Intelligence29(2014)79-92, Elsevier.

[29] Twan Dollevoet, Francesco Corman, Andrea D’Ariano, Dennis Huisman "An iterative optimization framework for delay management and train scheduling" Springer Science+ Business Media New York 2013.

[30] Özgür Yalçınkaya, G. Mirac Bayhan “A feasible timetable generator simulation modelling framework for train scheduling problem", Simulation Modelling Practice and Theory 20 (2012) 124-141 Elsevier.

[31] P. Tormos, A. Lova, F. Barber, L. Ingolotti, M. Abril, and M.A. Salido, "A Genetic Algorithm for Railway Scheduling Problems" Studies in Computational Intelligence (SCI) 128, 255-276 (2008),Springer-Verlag Berlin Heidelberg 2008.

[32] Antonio Lova, Pilar Tormos, Federico Barber, Laura Ingolotti, Miguel A. Salido, and Monsterrat Abril Intelligent Train Scheduling on a High-Loaded, Railway Network, LNCS 4359, pp. 219-232, 2007. SpringerVerlag Berlin Heidelberg 2007.

[33] David Cancaa, Alejandro Zarzo, Encarnación Algaba, Eva Barrena "Confrontation of Different Objectives in the determination of train Scheduling" Procedia Social and Behavioral Sciences 20 (2011) 302-312,Elsevier.

[34] Francesco Corman, Andrea D'Ariano ,Ingo A. Hansen , Dario Pacciarelli "Optimal multi-class rescheduling of railway traffic Journal of Rail Transport" Planning \& Management 1 (2011), 14-24 ,Elsevier.

[35] Andrea D'Ariano, Marcella Samà, Paolo D'Ariano, Dario Pacciarelli, "Evaluating the applicability of advanced techniques for practical real-time train scheduling" 17th Meeting of the EURO Working Group on Transportation, EWGT2014, 2-4 July 2014, Sevilla, Spain, Elsevier.

[36] Valentina Cacchiani, Dennis Huisman , Martin Kidd , Leo Kroon, Paolo Toth ,Lucas Veelenturf, Joris Wagenaar, "An overview of recovery models and algorithms for real-time railway rescheduling, Transportation Research Part B 63 (2014) 15-37 Elsevier

[37] Tomii Norio, Tashiro Yoshiaki, Tanabe Noriyuki, Hirai Chikara, and Muraki Kunimitsu, "Train Rescheduling Algorithm Which Minimizes Passengers'
Dissatisfaction", LNAI 3533, pp. 829-838, 2005, Springer-Verlag Berlin Heidelberg 2005.

[38] Selim Dündar, Ismail Sahin, “Train re-scheduling with genetic algorithms and artificial neural networks for single-track railways", Transportation Research Part C 27 (2013) 1-15.

[39] M. Almodo' var, R.Garc1'a-Ro' denas, "On-line reschedule optimization for passenger railways in case of emergencies", Computers \&Operations Research 40 (2013) 725-736, Elsevier.

[40] Juan A. Mesa, Francisco A. Ortega, Miguel A. Pozo and Justo Puerto, “ Rescheduling Railway Timetables in Presence of Passenger Transfers Between Lines Within a Transportation Network",

Computer-based Modelling and Optimization in Transportation, Advances in Intelligent Systems and Computing 262, Springer International Publishing Switzerland 2014.

[41] A.R. Albrecht, D.M.Panton, D.H.Lee, "Rescheduling rail networks with maintenance disruptions using Problem Space Search", Computers \& Operations Research 40(2013)703-712, Elsevier.

[42] Enrique Castillo, Inmaculada Gallego, José María Ureña, José María Coronado, "Timetabling optimization of a single railway track line with sensitivity analysis", springer 2008

[43] L. Ingolotti, A. Lova , F. Barber, P. Tormos, M.A. Salido, and M. Abril, "New Heuristics to Solve the "CSOP" Railway Timetabling Problem", LNAI 4031, pp. 400-409, 2006. Springer-Verlag Berlin Heidelberg 2006.

[44] Sundravalli Narayanswami, Narayan Rangaraj, "Scheduling and Rescheduling of Railway Operations: A Review and Expository Analysis" springer 2011

[45] A. Jamili , M.A.Shafia , S.J.Sadjadi, R.TavakkoliMoghaddam, "Solving a periodic single-track train timetabling problem by an efficient hybrid algorithm" Engineering Applications of Artificial Intelligence 25 (2012)793-800, Elsevier.

[46] Enrique Castillo, Inmaculada Gallego , José María Ureña, José María Coronado, "Timetabling optimization of a mixed double- and single-tracked railway network", Applied Mathematical Modelling 35 (2011) 859-878, Elsevier.

[47] Feng Jiang, Shaoquan Ni, and Daben Yu "Train Timetable Forming Quality Evaluation: An Approach Based on DEA Method", Springer International Publishing Switzerland 2015, Model. Comput. \& Optim. In Inf. Syst. \& Manage. Sci., 437. Advances in Intelligent Systems and Computing.

[48] Christian Liebchen and Rolf H. M"ohring "The Modeling Power of the Periodic Event Scheduling Problem: Railway Timetables - and Beyond", LNCS 4359, pp. 3-40, 2007, Springer-Verlag Berlin Heidelberg 2007

[49] Ruhe Xie", Guixiu Luo"\& Zhongren Peng, "Optimization to the Train Operating Schedule of Intercity High Speed Railway", 2009 IEEE. 
[50] Y. Larroche, R. Moulin and D. Gauyacq, "sepia: a realtime expert system that automates train route management", Control Eng. Practice, Vol. 4, No. 1, pp. 27-34, 1996 Elsevier Science Ltd.

[51] Tiberiu Letia, Mihai Hulea and Radu Miron, "Distributed Scheduling for Real-Time Railway Traffic Control", Proceedings of the International Multi conference on Computer Science and Information Technology pp. 679685 .

[52] Cheng Wang, “An agent-based Monitoring Architecture for CTCS-3 Distributed Simulation System"

[53] Jingdong Sun, Yao Wang, and Shan Wang, "Distributed Hierarchical Control for Railway Passenger-Dedicated Line Intelligent Transportation System Based on MultiAgent”, LNCS 6146, pp. 252-259, 2010, SpringerVerlag Berlin Heidelberg 2010

[54] LIU Zi-yu, HUANG Lei, "Research on the IDSS of High-speed Railway Based on Multi-agent”, 2007 International Conference on Management Science \& Engineering (14th), August 20-22, 2007.

[55] Chaozhe JIANG, Jin YANG, Jixue YUAN, "Development and Sustainable Strategies of RITS in China", Proceeding of the IEEE, International Conference on Automation and Logistics, 'Chongqing, China, August 2011.

[56] Ping L1, Li-Min JIA, A-Xin NIE, "Study on Railway Intelligent Transportation System Architecture".

[57] M. Clarke, C. J. Hinde, M. S. Withall, T. W. Jackson, I. W. Phillips, S. Brown, and R. Watson, "Allocating Railway Platforms Using A Genetic Algorithm", Research and Development in Intelligent Systems XXVI ,Springer-Verlag London Limited 2010.

[58] Petrica C. Pop, Camelia M. Pintea, and Corina Pop Sitar, "An Ant-Based Heuristic for the Railway Traveling Salesman Problem" Evo Workshops 2007, LNCS 4448, pp. 702-711, 2007. Springer-Verlag Berlin Heidelberg 2007.

[59] Chao WEN "Train operation conflict management research status of high-speed railways" Springer Science + Business Media, LLC 2011.

[60] Maria Pia Fanti, Alessandro Giua, Carla Seatzu , “A deadlock prevention method for railway networks using monitors for colored Petri nets", 2003 IEEE.

[61] Jacek Zak, "Decision Support Systems in Transportation Handbook on Decision Making", ISRL 4, pp. 249-294. Springer-Verlag Berlin Heidelberg 2010

[62] Antoine lemortellec, Joffrey Clarhaut, yvessallez , Thierry Berger, damientrentesaux "Embedded holonic fault diagnosis of complex transportation systems" Engineering Applications of Artificial Intelligence 26 (2013) 227-240 Elsevier.

[63] Andrew P. Moran, Annegret H. Thieken, Andreas Schöbel, and Christian Rachoy "Documentation of Flood Damage on Railway Infrastructure Data and Mobility", AISC 81, pp. 61-70, Springer-Verlag Berlin Heidelberg 2010.
[64] Liujiang Kang, Jianjun Wua, Huijun Sun, Xiaoning Zhu , Ziyou Gao "A case study on the coordination of last trains for the Beijing subway network" Transportation Research Part B 72 (2015) 112-127, Elsevier.

[65] Christoph Bergmeir, Gregorio S'ainz, Carlos Martınez Bertrand, and Jose Manuel Benitez "A Study on the Use of Machine Learning Methods for Incidence Prediction in High-Speed Train Tracks" LNAI 7906, pp. 674-683, 2013. Springer-Verlag Berlin Heidelberg 2013

[66] Lixing Yang, Xuesong Zhou, Ziyou Gao "Rescheduling trains with scenario-based fuzzy recovery time representation on two-way double-track railways" Springer-Verlag 2012

[67] Tao Wu, Yusong Yan, and Xi Chen1, "Reduction of Power Consumption in Wireless Sensor Networks for Railway Disaster Prevention and Safety Monitoring System", AISC 180, pp. 983-991. Springer-Verlag Berlin Heidelberg 2013.

[68] Dimitrios A. Tsamboulas , George K. Mikroudis "TRANS-POL: A mediator between transportation models and Decision makers' policies" Decision Support Systems 42 (2006) 879- 897 Elsevier.

[69] Yu-Jun Zheng, Hai- Feng Ling, Hai-heshi, Hai-Song Chen, Sheng-yongchen, "Emergency railway wagon scheduling by hybrid biogeography-based optimization", Computers \& Operations Research 43 (2014), 1-8, Elsevier.

[70] BY SIR WILLIAM CROOKES, "The wheat supply of the world the latest achivments of science and the position of phychical research", THE LANSET SEPTEMBER 101898.

[71] Paola Pellegrini, Grégory Marlière, Joaquin Rodriguez, "Optimal train routing and scheduling for managing traffic perturbations in complex junctions", Transportation Research Part B 59 (2014) 58-80, Elsevier.

[72] Decision Support Systems and Intelligent Systems, Efraim Turban and Jay E. Aronson, 6th ed, Copyright 2001, Prentice Hall, Upper Saddle River, NJ.

[73] Artificial intelligence second edition, Elaine rich, Kevin Knight, Tata Mcgraw Hilll edition 2006.

[74] Francesco corman, "real time railway traffic management: dispatching in complex, large and busy railway network, 2010, ISBN 978-90-5584-133-2.

[75] Vincent van der Vlies, "Rail Transport Risks and Urban Planning: Solving deadlock situations between urban planning and rail transport of hazardous materials in the Netherlands". ISBN 978-90-5584-149-3, 2011.

[76] Chris w. Janczura, "Modelling and analysis of railway network control logic using coloured petri nets, 1998.

[77] Indian Railways, Lifeline of the nation, A White Paper, February 2015, Government of India, Ministry of Railways, NEW DELHI. 